\title{
Death Rates and US Presidential Voting
}

\author{
Lee Goldman, MD MPH', ${ }^{1,2}$, Maribel P. Lim, MS ${ }^{7}$, Qixuan Chen, PhD ${ }^{2}$, Peng Jin, MS ${ }^{7}$, \\ Peter Muennig, MD MPH ${ }^{2}$, and Andrew Vagelos, $M A^{\top}$
}

${ }^{1}$ Vagelos College of Physicians and Surgeons, Columbia University, New York, NY, USA; ${ }^{2}$ Mailman School of Public Health, Columbia University, New York, NY, USA.

J Gen Intern Med 35(3):936

DOI: $10.1007 / \mathrm{s} 11606-019-05574-7$

(C) Society of General Internal Medicine 2019

$\mathrm{T}_{\text {Fis }}$ o the Editors:

First, we stand fully by our analyses and interpretations. Second, although we are pleased to see students, especially two of our recent master's graduates, read the literature and engage in important issues, scientific integrity demands a response guided by our first statement.

Our findings explicitly were based on how changes in countywide metrics affected changes in presidential voting between 2008 and $2016^{1}$ because changes in these metrics are often more telling than the metrics themselves ${ }^{2}$. However, analogous findings have been reported based on static metrics ${ }^{3,4}$.

A careful reading of our article shows that we emphasized repeatedly that correlation does not prove causality and explicitly acknowledged that county-wide data, which are the most granular that are reliably available, do not represent individuals. We also indicated that exit polling and other attempts to obtain individual voting data are notoriously unreliable.

Although the opioid epidemic has had a devastating impact on communities throughout the USA, our analysis showed that what are termed "deaths of despair" (deaths due to suicides, drugs, and alcohol) represented only about $5 \%$ of deaths in 2016 and did not add independent information when death rates from other causes were included. A careful reading of our manuscript also shows that the hypothetical effect of lifestyle changes on voting results was made by others ${ }^{5}$ and cited by us to emphasize that blunted reductions in death rates are more a reflection of obesity, diabetes, heart disease, smoking-related diseases, and the like than of the more highly publicized deaths of despair. Although we also hope that public health interventions and health insurance would narrow these gaps, counties supporting President Trump had poorer health outcomes than other counties despite similar rates of health insurance.

We showed that the Republican gain from 2008 to 2016 was independently correlated with changes in county-wide death rates even after adjusting for the economic and demographic characteristics of the so-called "left behind" voter. We do not dispute President Trump's appeals to racism, but the Democratic party's 2008 African American candidate outperformed its 2016 white candidate.

The strategies that political campaigns and legislatures may use to capitalize on the status, change in status, hopes, and fears of the populace may be estimated by pundits, polls, or even political scientists. By comparison, our analyses of demographic and epidemiologic facts were specifically designed to assess the realities on which such campaign strategies might be based.

Corresponding Author: Lee Goldman, MD MPH; Vagelos College of Physicians and Surgeons, Columbia University, New York, NY, USA (e-mail: lgoldman@columbia.edu).

\section{REFERENCES}

1. Goldman L, Lim MP, Chen $\mathbf{~}$, Jin P, Muennig P, Vagelos A. Independent relationship of changes in death rates with changes in US presidential voting. J Gen Intern Med. 2019;34(3):363-71.

2. Easterlin RA. Explaining happiness. Proc Natl Acad Sci U S A. 2003; 100(19):11176-83.

3. Bor J. Diverging life expectancies and voting patterns in the 2016 US presidential election. Am J Public Health. 2017;107(10):1560-2.

4. Wasfy JH, Stewart C, 3rd, Bhambhani V. County community health associations of net voting shift in the 2016 U.S. presidential election. PLoS One. 2017;12(10):e0185051.

5. Illness as indicator: local health outcomes predict Trumpward swings. Economist. 2016. http://www.economist.com/node/21710265. Accessed July 30, 2019.

Publisher's Note Springer Nature remains neutral with regard to jurisdictional claims in published maps and institutional affiliations. 